\title{
An assessment of interrater reliability of the ASA physical status classification in pediatric surgical patients
}

\author{
JACQUELINE RAGHEB MD FFARCSI, SHOBHA MALVIYA MD, \\ CONSTANCE BURKE BSN RN AND PAUL REYNOLDS MD \\ Department of Anesthesiology, Section of Pediatrics, University of Michigan, Ann Arbor, MI, \\ USA
}

\section{Summary}

Background: The American Society of Anesthesiologists physical status classification (ASA-PS) is used worldwide by anesthesia providers as an assessment of the preoperative physical status of patients. This assessment score has been inconsistently assigned by anesthesia providers among adult surgical patients. This study tested the reliability of assignment of ASA-PS classification among pediatric anesthesia providers.

Methods: A postal questionnaire was sent to a randomly selected sample of full members of the Society of Pediatric Anesthesiologists. Participants were asked to assign ASA-PS for 10 clinical case scenarios chosen from regular pediatric surgical cases at the investigators' institution.

Results: The response rate to our mailing was $54 \%$. There was a moderate overall agreement among pediatric anesthesia providers in assigning ASA-PS for pediatric surgical patients (exact agreement $40.5-78.6 \% ; \kappa=0.479)$. Exact agreement improved for combined ASA classifications of I and II (83\%), and III and IV (95\%).

Conclusion: These findings suggest a moderate agreement among pediatric anesthesia providers in assigning ASA-PS classification to selected pediatric case scenarios. Most disagreement, however, represented a tendency of outside care providers to assign a higher ASA physical status for cases. Furthermore, agreement was excellent for low risk (i.e. ASA I and II) as well as high risk (ASA III and IV) cases.

Keywords: ASA physical status classification; pediatric; surgical patients; reliability 


\section{Introduction}

The American Society of Anesthesiologists physical status (ASA-PS) classification Table 1. (1) is used worldwide by anesthesia providers as an assessment of the preoperative physical status of patients (2). This score has been used in policy making, performance evaluation, resource allocation, reimbursement of anesthesia service and frequently cited in clinical research (3). Previous studies in adult patients have reported that anesthesia providers have frequently assigned inconsistent ASA scores to the same patient (3-5).

The present study was therefore undertaken to evaluate the interrater reliability of the ASA-PS classification scoring system among pediatric anesthesia providers. We hypothesized that there would be adequate agreement between pediatric anesthesia providers in assigning ASA-PS for routine pediatric surgical patients.

\section{Methods}

After Institutional Review Board approval, we surveyed a random sample of members of the Society of Pediatric Anesthesiologists (SPA) to evaluate the interrater reliability of the ASA physical status classification in pediatric surgical patients. A questionnaire with 10 real pediatric case scenarios (Appendix 1) chosen from our regular surgical population was developed. The questionnaire contained items eliciting demographics, and number of years in practice, but it contained no identifying information. The case scenarios were reviewed by five anesthesiologists in our department, and an ASA-PS

Table 1

ASA physical status classification $(1961)^{a}$

\begin{tabular}{ll}
\hline I & A normal healthy patient \\
II & A patient with mild systemic disease \\
III & A patient with severe systemic disease \\
& that limits activity but not incapacitating \\
IV & A patient with incapacitating disease \\
& that is a constant threat to life \\
V & A moribund patient not expected to survive \\
& 24 h with or without surgical operation \\
E & In the event of an emergency operation, \\
& an E is placed after the roman numerical \\
\hline
\end{tabular}

${ }^{a}$ Adapted from the American Society of Anesthesiologists. classification was assigned to each. Each of these members had been a pediatric anesthesiologist practicing for more than 10 years. The ASA score assigned more frequently by the anesthesiologists for each case scenario was considered to represent consensus agreement for that scenario. The questionnaire was mailed to the selected sample who were asked to complete the survey, assigning ASA-PS score for each of these scenarios, and return the survey in an enclosed preaddressed postage paid envelope.

Data were analyzed with SPSS software (vs 13.0; SPSS, Inc., Chicago, IL, USA). Comparisons between raters' ASA-PS levels were made using chi-square and Fisher's exact tests where appropriate. The level of agreement is described as percent agreement, and was evaluated with kappa statistics. Data are presented as $n(\%)$ or mean \pm SD as applicable, and $P$-values of $<0.05$ were accepted as significant. Kappa values of $>0.41$ were considered acceptable agreement.

\section{Results}

One hundred questionnaires were mailed, of which 54 were completed and returned. Three surveys were returned for undeliverable address. This resulted in a response rate of $56 \%$. Of the respondents, $94.1 \%$ were anesthesiologists with an MD degree, and $2 \%$ had a DO degree. The mean age of respondents was 46.6 years, $23.5 \%$ had between 10 and 15 years of practice, and $23.5 \%$ had more than 20 years of practice.

Exact agreement for each ASA-PS classification is presented in Table 2. There was a moderate overall agreement between respondents $(\kappa=0.471)$. When cardiac cases were excluded, the exact agreement for ASA III cases improved from $64.2 \%$ to $86.1 \%$, with an overall improvement in agreement across all

Table 2

Agreement between respondents' and standard raters' ASA-PS Assignment

\begin{tabular}{lcccc}
\hline \multirow{2}{*}{$\begin{array}{l}\text { Consensus panel } \\
\text { assignment }\end{array}$} & \multicolumn{4}{c}{ Survey respondents assignment (\%) } \\
\cline { 2 - 5 } & ASA I & ASA II & ASA III & ASA IV \\
\hline ASA I & $68(40.5)$ & $87(51.8)$ & $13(7.7)$ & $0(0.0)$ \\
ASA II & $3(2.7)$ & $88(78.6)$ & $21(18.8)$ & $0(0.0)$ \\
ASA III & $0(0.0)$ & $13(7.9)$ & $106(64.2)$ & $46(27.9)$ \\
ASA IV & $0(0.0)$ & $0(0.0)$ & $43(38.7)$ & $68(61.3)$ \\
\hline
\end{tabular}


classifications $(\kappa=0.524)$. There was also improved agreement when we combined ASA I and II (87.9\%), and ASA III and IV (95.3\%). The overall agreement in assignment of ASA-PS classification did not differ significantly between raters in private settings compared with those in academic settings $(\kappa=0.454)$. Lastly, the interrater agreement for assignment of nonemergency status was $87.8 \%$, and $83 \%$ for emergency status.

\section{Discussion}

Despite the simplicity and ease of use of ASA classification, there has been a documented inconsistency in ASA assignment for adult patients by different anesthesia providers (3-5). Our results, however, show that in a sample of pediatric anesthesia providers, there was an adequate agreement in their assignment of ASA-PS. These findings suggest that, in general, assignment of ASA-PS by pediatric anesthesiologists is reliable.

Notably when children with congenital heart disease were excluded from the statistical analysis, agreement in assigned ASA scores improved slightly. This finding could perhaps be explained in part by a tendency of providers not routinely involved in the care of children with cardiac comorbidity, to overestimate the physical status of these patients. Conversely, providers who routinely anesthetize such patients may have underestimated the physical status and therefore assigned a lower ASA score. The improvement of overall agreement between anesthesia providers for combined ASA I and II cases and ASA III and IV cases reflects a better agreement of overall health status of patients, where classically ASA I and II patients are regarded as 'fit' or low-risk patients while ASA III and IV patients are usually regarded as 'unfit' or high-risk patients.

Interestingly, the interrater agreement for the assignment of emergent status was only $84.3 \%$. We would have expected that the agreement would have been higher for assignment of emergent status across settings. On further analysis, the case scenario which provoked the most inconsistency was case number 10 , where there was only $72 \%$ agreement that the case was nonemergency. This may perhaps be explained by the ongoing controversy whether pyloric stenosis is a medical or a surgical emergency (6).
It is interesting to note that most of the disagreement in assignment of ASA-PS status for our cases was generated by a tendency of survey respondents to assign a higher ASA-PS score to patients. Although not strictly intended as an assessment of risk, studies have demonstrated a correlation between ASA-PS and outcome of anesthesia (7). This would suggest a need to be particularly vigilant when caring for patients with a higher classification. Our finding that respondents frequently assigned a higher ASA-PS to patients compared with our assignments, may therefore, demonstrate a tendency of many pediatric anesthesiologists to err on the conservative side.

Interpretation of our data are subject to the following limitations. As we surveyed the members of the SPA only, our results cannot be generalized to all anesthesiologists. Furthermore, we did not include a case scenario for a patient with ASA V classification. Therefore, interrater reliability of this classification was not evaluated.

Lastly, the survey methodology used in this study precluded the respondents from soliciting additional patient's medical information. It remains unclear from our data if the ability to obtain additional information may have resulted in assignment of a different ASA status in some cases.

The moderate interrater reliability of ASA-PS classification assignment by pediatric anesthesia providers to common pediatric surgical patients suggests that this remains a reasonable method to provide a good estimate of anesthesia risk. The interrater agreement between such scores assigned by all anesthesia providers in select populations remains to be determined.

\section{References}

1 Saklad M. Grading of patients for surgical procedures. Anesthesiology 1941; 2: 281-284.

2 American society of Anesthesiologists. New classification of physical status [editorial]. Anesthesiology 1963; 24: 111.

3 Haynes SR, Lawler PGP. An assessment of consistency of ASA physical status classification allocation. Anesthesia 1995; 50: 195199.

4 Ranta S, Hynynen M, Tammisto T. A survey of the ASA physical status classification: significant variation in allocation among Finnish anesthesiologists. Acta Anesthesiol Scand 1997; 41: 629-632.

5 Aronson WL, McAuliffe MS, Miller K. Variability in the American society of Anesthesiologists Physical status classification scale. AANA J 2003; 71: 265-274. 
6 Bissonnette B, Sullivan PJ. Pyloric stenosis. Can J Anaesth 1991; 38: $668-676$.

7 Tiret L, Hatton F, Desmonts JM et al. Prediction of outcome of anesthesia in patients over 40 years: a multifactorial risk index. Stat Med 1988; 7: 947-954.

Accepted 7 January 2006

\section{Appendix 1. Case scenarios}

\section{Case 1}

A 3-year-old girl was scheduled for emergency replacement of jejunal feeding tube.

Medical history significant for hypoplastic left heart syndrome, chronic subdural hematomas and reflux esophagitis. The patient had undergone a Norwood procedure at 1 month of age and a Nissen fundoplication at 2 years of age. Her parents described cyanotic spells on crying and she was noted to be oxygen dependent. Her oxygen saturation was $70-80 \%$, on room air.

\section{Case 2}

A 9-year-old patient was scheduled for strabismus repair. She had a past history of bronchial asthma necessitating hospitalization at 1 year of age for oxygen therapy. Currently, the asthma was well controlled on nebulized metered treatments. The patient had a history of allergy to sulfonamides. Physical examination was, otherwise, unremarkable.

\section{Case 3}

A 5-year-old patient was scheduled for emergency laparotomy for suspected bowel perforation. The patient had a history of non-Hodgkin's lymphoma treated with chemotherapy and radiation. Last chemotherapy treatment was 1 week prior to surgery.

\section{Case 4}

A 14-year-old male patient weighing $65 \mathrm{~kg}$ was scheduled for anterior and posterior spinal fusion. History of muscular dystrophy with progressive scoliosis. Preoperative pulmonary function tests revealed moderate degree of restrictive lung disease.

\section{Case 5}

A 16-year-old female patient weighing $70 \mathrm{~kg}$ was scheduled for urinary bladder reconstruction. She had a history of congenital urological abnormalities requiring multiple reconstructive surgeries in the past. Additionally, she had a history of idiopathic cardiomyopathy with estimated ejection fraction at $25 \%$. Potentially fatal arrhythmias required insertion of permanent pacemaker and implantable defibrillator.

\section{Case 6}

A 3-month-old baby weighing $5 \mathrm{~kg}$ born at 28 weeks gestational age was scheduled for bilateral inguinal hernia repair. The baby had required mechanical ventilation in the neonatal ICU for 3 weeks postdelivery. Physical examination was unremarkable.

\section{Case 7}

A 6-month-old male infant weighing $7 \mathrm{~kg}$ was admitted for repair of cleft palate. The baby who was born at full term had respiratory difficulties requiring nasal CPAP for 3 weeks postbirth. The clinical diagnosis was of Pierre Robin sequence, with no other significant congenital anomalies.

\section{Case 8}

A 6-year-old patient weighing $20 \mathrm{~kg}$ with posttonsillectomy bleeding was scheduled for exploration and hemostasis. She had adenotonsillectomy the previous morning. No relevant past medical history and no allergies.

\section{Case 9}

A 38 week gestational age infant weighing $3.6 \mathrm{~kg}$ with an antenatal diagnosis of hypoplastic left heart syndrome scheduled for staged repair.

\section{Case 10}

A 1 day old, full-term male infant was scheduled for pyloromyotomy for pyloric stenosis. His physical examination was otherwise normal. 